\title{
Análisis de dominios científicos de la investigación de la traducción de obras de Mo Yan en China a través de Citespace
}

\section{Knowledge Mapping Analysis of Translation of Mo Yan's works in China using Citespace}

\author{
ZHAO YUAN YUAN \\ Zhao.yuanyuan737@gmail.com \\ Universidad de Huelva
}

Fecha de recepción: 12 de diciembre de 2020

Fecha de aceptación: 18 de enero de 2021

\begin{abstract}
Resumen: El presente trabajo tiene el fin de dar a conocer, con la aplicación de Citespace V.5.3.R7, herramienta para analizar registros bibliográficos de publicaciones relevantes, la tendencia del estudio traductológico de las obras de Mo Yan en China durante el período del 1999 al 2019. Por ello, se recopila una base de dato bibliográfico compuesta por 1,529 artículos académicos al respecto de la plataforma CNKI. Basándose en redes Pathfinder y Pruning sliced network, se revelan las concentraciones temáticas de referencias cocitadas, las líneas de investigación por palabras clave y clústeres, se analiza la distribución cuantitativa de las publicaciones, se destacan las revistas, instituciones y autores clave por medio de grafo y datos de CNKI. De acuerdo con los datos sacados, se harán contribuciones para profundizar el estudio traslativo de obras de Mo Yan.
\end{abstract}

Palabras clave: Mo Yan, Bibliometría, CNKI, Traducción, Citespace

Abstract: The aim of this study is to promote the trend of translation studies of Mo Yan's works in China from 1999 to 2019 by applying Citespace (V.5.3.R7), a tool for analyzing the literature records of relevant publications. Therefore, a bibliographic database of 1,529 academic articles on CNKI platform is being established. Based on the data of pioneers and experimental sliced networks, the concentration of reference materials by topic, research lines and pamphlets by keywords, quantitative distribution analysis of publications, and highlighting key journals, institutions, and authors through CNKI graphics and data. It provides a reference for the study of the translation of Mo Yan's works and the development of the discipline.

Keywords: Mo Yan, Bibliometrics, CNKI, Translation, Citespace 


\section{INTRODUCCIÓN}

Mo Yan, ganador del Premio Nobel de Literatura de 2012, es uno de los más destacados escritores contemporáneos de China. Comenzó a publicar en 1981 y lleva en su haber una importante obra narrativa, entre ellas, Lluvia en una noche de primavera, El rábano transparente, El sorgo rojo, así como, en 2011, Rana con las que obtuvo el Premio Literatura Mao Dun ${ }^{1}$ de 2011 y el Nobel de 2012. En China, con la difusión de las obras de Mo Yan, se van aumentando los estudios con enfoques en el ámbito literario y traslativo. El presente estudio intenta dar a conocer de forma objetiva la situación de las investigaciones de sus obras traducidas con aproximaciones descriptivas y cuantitativas. Para llegar a este fin propuesto, recurrimos a la bibliometría que nos hace entender las disciplinas de investigación en base a la aplicación de modelos y métodos cuantitativos, tales como el análisis de redes, los enlaces y sus números correspondientes, fuerza de enlace dentro de las redes de co-ocurrencia, co-citación, coautoría y vínculo bibliográfico (Martínez-Toro et al, 2019, p. 313). La bibliometría atiende cinco grandes grupos de fenómeno y su análisis: primero, en el análisis de citación para individuos, instituciones, países y disciplinas (White y Mccain,1998); segundo, el análisis de co-citación (Small,1973) para el mapeo de países, individuos, escuelas de pensamiento, etc.; tercero, la productividad científica (Sink, Tuttle, y Devries, 1984) por medio de análisis de impacto (Narin,1987) de individuos, países e instituciones; cuarto, el análisis de productos específicos como artículos o patentes, basados en características del autor, patrones de citación y característica longitudinales (Terry, 1994) y quinto, coocurrencia de palabras, referencias, etc. (Naghizadeh, Elahi, Manteghi, Ghazinoory, y Ranga, 2015).

\section{Objetivo del trabajo}

El presente trabajo tiene el objetivo de visualizar y conocer la evolución de la investigación de las obras de Mo Yan traducidas al español. Por ello, se han investigado las siguientes preguntas relacionadas:

- Presentar datos generales sobre esta área de investigación.

- Identificar las principales líneas de investigación.

- Conocer el cambio repentino producido en la línea de una investigación.

- Localizar a los autores e instituciones con mayor influencia dejada en el ámbito referente.

\footnotetext{
${ }^{1}$ El Premio de Literatura Mao Dun está patrocinado por la Asociación de Escritores Chinos y se estableció sobre la base de la voluntad del Señor Mao Dun para alentar la creación de novelas sobresalientes y promover la prosperidad de literatura china. Es uno de los premios de honor más importantes de China.
} 
- Detectar tendencias emergentes de investigación.

\section{MATERIALES Y MÉtOdOS}

Con el fin de establecer la evolución de la investigación, se destacan los métodos y herramientas bibliométricas.

La metodología consta de tres frases, 1. recolección de artículos; 2. procesamiento de datos; 3 . análisis bibliométrico.

\subsection{Datos recopilados}

Todos los datos que se analizan en el estudio proceden de $\mathrm{CNKI}^{2}$. $\mathrm{El}$ período de análisis comprende las descargas de las publicaciones de CNKI desde 1999 hasta principios de 2020. Cuando colocan palabras clave "Mo Yan" y "Traducción" en la barra de búsqueda y eligen "Topic" (esta opción incluye el título, resumen, palabras clave, país) como el elemento de recuperación y el resultado es de 1529 descargas realizadas que se clasifican en:

- Artículos: 907.

- TFM y tesis: 454 .

- Congresos: 33.

- Periódicos: 101.

- Libros: 9.

- Otras publicaciones: 28.

(La última fecha de consulta: el 15 de febrero de 2020)

\subsection{Métodos}

En 1955, se publicó Citation Indexes for Science: A New Dimension in Documentation through Association of ideas de Eugene Garfield quien planteó que podíamos averiguar el desarrollo de una línea de investigación tras realizar la indización por citas, por la que se formó el paradigma de la metodología de la bibliometría (Garfield E, 1955). En otras palabras, esto significa que "el uso de las citas en las publicaciones académicas se articula a través de fórmulas matemáticas con la ayuda de un ordenador para relevar los enlaces, las relaciones académicas y la red de conocimiento" (Chen. 2014, p.15).

El análisis de dominios científicos recurre al análisis de redes sociales para comprender la información a través de grafo, lo que permite visualizar el conocimiento científico al definir una estructura social donde los nodos se representan por individuos o documentos y los enlaces son el conocimiento

2 CNKI, China National Knowledge Infrastructure. 
que intercambian dichos nodos (Börner, Chen, y Boyack, 2003, p. 7). En la actualidad, se apoya en técnicas de visualización.

En cuanto a la visualización de dominio científico. Se determinó las unidades de medida (co-citación, emparejamiento bibliográfico, co-ocurrencia y co-auditoría) tomadas para la la visualización de dominios científicos, con la finalidad de observar, y analizar las relaciones existentes entre cada una de las unidades de análisis (documentos, autores, palabras clave, instituciones, entre otros) con el resto de sus integrantes (Guerrero Chanduví, Dante A. M. y La Rosa Lama, Gerson, 2014).

Como métodos se implementan el análisis de co-citación y coocurrencia de palabras, los cuales sirven como medida de relación entre documentos (Small, 1973).

La co-citación como medida de relación establecida por los autores que citan, se define como la frecuencia con la que dos trabajos de literatura anterior se citan juntos en la literatura posterior (Small, 1973). La fuerza de co-cita mide el grado de relación entre los documentos (Koehler, 2001). La frecuencia de co-citación, corresponde al número de veces con la que se citan dos documentos de manera simultánea (Liu, Yin, Liu y Dunford, 2015). La unidad de análisis de los estudios de este tipo comprende autores, fuentes y referencias o artículos citados (van Eck y Waltman, 2010). "Cuanto mayor sea el vínculo de co-citación entre ambos, mayor será la proximidad de los autores y las revistas derivados de esas relaciones" (Olmeda-Gómez, Perianes-Rodríguez, 2019, p. 4). Un análisis de co-citación es útil para identificar la estructura de un campo de investigación, así como los cambios ocurridos. Los mapas de co-citación de documentos sirven para identificar las especialidades de un tema. Por otro lado, un análisis de co-citación de autores, permiten representar la estructura intelectual y social de un campo de investigación (Hyunjung, 2012).

A ello se suma, según Martínez-Toro et al. (2019, p. 366) "La coocurrencia de palabras clave indica la relación de proximidad entre dos o más palabras clave dentro de un documento, se representa mediante las gráficas de redes de enlace entre términos". El análisis de palabras clave es de amplia difusión dentro del análisis de contenido central de la literatura de investigación (Naghizadeh et al., 2015). Los análisis de co-ocurrencia revelan patrones y tendencias en una disciplina específica midiendo la fuerza de la asociación de los términos representativos de publicaciones relevantes producidas en el área (Ding, Chowdhury y Foo, 2001). 


\section{ANÁLISIS BIBLIOGRÁFICO}

\subsection{Citespace}

Las herramientas bibliométricas se han utilizado ampliamente en la escritura de revisión académica, lo que puede evitar en gran medida la influencia de la subjetividad de los investigadores cuando sacan las conclusiones. En el artículo presente se analiza la herramienta bibliométrica CiteSpace para hacer la revisión de la investigación de la traducción de las obras de Mo Yan en China (CiteSpace es una herramienta específica para el análisis de citas).

CiteSpace II —programa desarrollado por Chaomei Chei (2006)— es una aplicación gratuita de Java, que se encuentra disponible para la visualización y análisis de data sobre dominio de conocimiento en la que representa sus nodos y frecuencias. La función de este programa es identificar las relaciones entre los autores que más obras producen, los términos más utilizados por los autores, documentos y revistas más citados (Adilson LP, José Antonio MG, 2009). Utilizando esta aplicación se genera un análisis sobre la cantidad, instituciones, autores y líneas de investigación a lo largo de, aproximadamente, 30 años.

\subsection{Establecimiento de Parámetros}

El atlas se presenta principalmente en forma de nodos y líneas, $\mathrm{N}=\mathrm{el}$ número de nodos, $\mathrm{E}=$ el número de líneas. El tamaño de los nodos refleja la frecuencia de referencias u ocurrencias de datos relevantes, las líneas indican la relación entre los nodos, y el grosor de las líneas entre nodos refleja la fuerza de los enlaces entre los datos (Yunong W; Huijir W; Zhexiao W; Bin $Z$; Burghard $C, 2019$ ). El efecto de clúster se midió utilizando modularidad y silueta (Yunong W; Huijir W; Zhexiao W; Bin Z; Burghard C,2019).

En cuanto al valor $Q$,

El valor $Q$ representa el grado de modularidad; $Q \geq 0.3$ significa que la modularización de la red es significativa y, a medida que aumenta el valor de $Q$, el efecto de clúster de la red mejora. La silueta (S) mide la homogeneidad de las redes; $S \geq 0.5$ significa que el resultado de la agrupación es razonable, y a medida que $S$ se acerca a 1, la homogeneidad de la red aumentará (Yunong W; Huijir W; Zhexiao W; Bin Z; Burghard C, 2019).

En el caso del trabajo de Mo Yan se han analizado y visualizado estas palabras clave empleando CiteSpace. El período elegido es entre 1999 y 2019 (20 años) y se ha dividido en subperíodos de 1 año. Las fuentes del clúster son title, abstract, autor, palabras clave y palabras clave plus. Elegimos el tipo de nudo keyword y se encontraron las 50 palabras clave más 
citadas a lo largo de cada subperíodo. Con esto, obtuvimos un grado de 187 nodos y 513 enlaces de co-citación con una densidad de red de 0,0653. $\mathrm{Q}=0.5071$ representa lo significativo de la modularización de la red de clústeres, $\mathrm{S}=0.5321$ representa una alta homogeneidad de las redes.

\subsection{Procedimientos e Indicadores}

\section{Burst}

Es el indicador de fuerza basado en la frecuencia significativa o la intensidad de la atención recibida por un determinado autor o revista en intervalo corto de tiempo. Se calcula respecto de otro momento similar en un ciclo temporal determinado (Mane; Börner, 2004). Es un indicador de orden superior para medir el impacto basado en la citación (Olmeda-Gómez, C; Perianes-Rodríguez, A, 2019).

\section{Silueta}

El valor de la silueta muestra la calidad de la configuración de los clústeres (Olmeda-Gómez, C; Perianes-Rodríguez, A, 2019). Cuanto mayor sea, más perfecta será la solución obtenida (Rousseeuw, 1987).

\section{Modularity}

Representa la medida en que una red se puede descomponer en múltiples componentes o módulos (Olmeda-Gómez, C; Perianes-Rodríguez, $A, 2019)$. Proporciona un valor matemático sobre la claridad de la descomposición de la red en clústeres (Newman, 2006; Chen; lbekweSanjuan; Hu, 2010).

\section{Centralidad}

Este indicador mide el número de caminos más cortos en una red que parten de todos los nodos hacia los demás, pasando por un nodo determinado (Freeman, 1977). "Centralidad es una métrica del nodo de la red que estipula la medida de la trayectoria más corta de la red, conocida también como intermediación" (Adilson Luis Pinto, José Antonio Moreiro González, 2009, p. 146). Centralidad describe el grado de centralización de la red, el valor más alto significa que la palabra ejerce el mayor control y tiene más influencia en su campo (Qiu y Zhang, 2011, p. 1443). La revista o el autor con valores más elevado de intermediación actúan como nodos conectores o conmutadores (Olmeda-Gómez, C y Perianes-Rodríguez, A, 2019, p. 5). Una palabra clave de mayor tamaño en Centralidad (Betweenness centrality $\geqq 0,1$ ) es un nodo clave que conecta con diferentes líneas de investigación o temas (Kong, 2019, p. 18). 


\section{Resultados}

\subsection{Distribución del número de publicaciones}

Después de la colección de los artículos publicados en la plataforma CNKI, en la figura 1 se refleja la evolución del número de publicaciones. Según la figura 1, se ve que la investigación inicia en el 1999 y existen dos virajes, el año 2011 y el 2014.

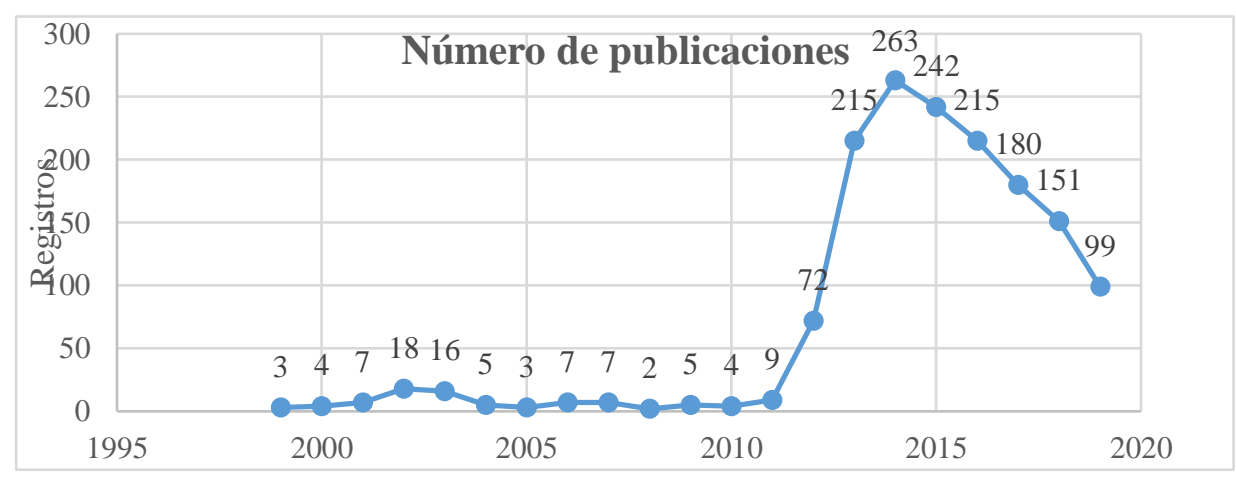

Figura 1. Evolución del número de publicaciones en la plataforma CNKI (1999-2019) utilizando como parámetros de búsqueda las secuencias "Mo Yan" y "traducción".

Fuente: Elaboración propia

En la figura 2 se plantea la tendencia de publicaciones entre 2014 y 2019, el número disminuye año tras año.

Si usamos como punto de partida el intervalo temporal de las publicaciones —en este caso, entre 1999 y 2011—; la cantidad de entradas sobre la investigación de las obras traducidas de Mo Yan arroja un resultado de 90 artículos. La mayoría de los documentos fueron posteriores al año 2011; el año con mayor número de publicaciones fue el 2014 (263 documentos); entre 2015 y 2019, el número de documentos registrados descendió año tras año (en la figura 2). Esto supone que, desde entonces, el número de investigaciones sobre su obra aumentó. Desde 2014, el número representa una tendencia de caída. 


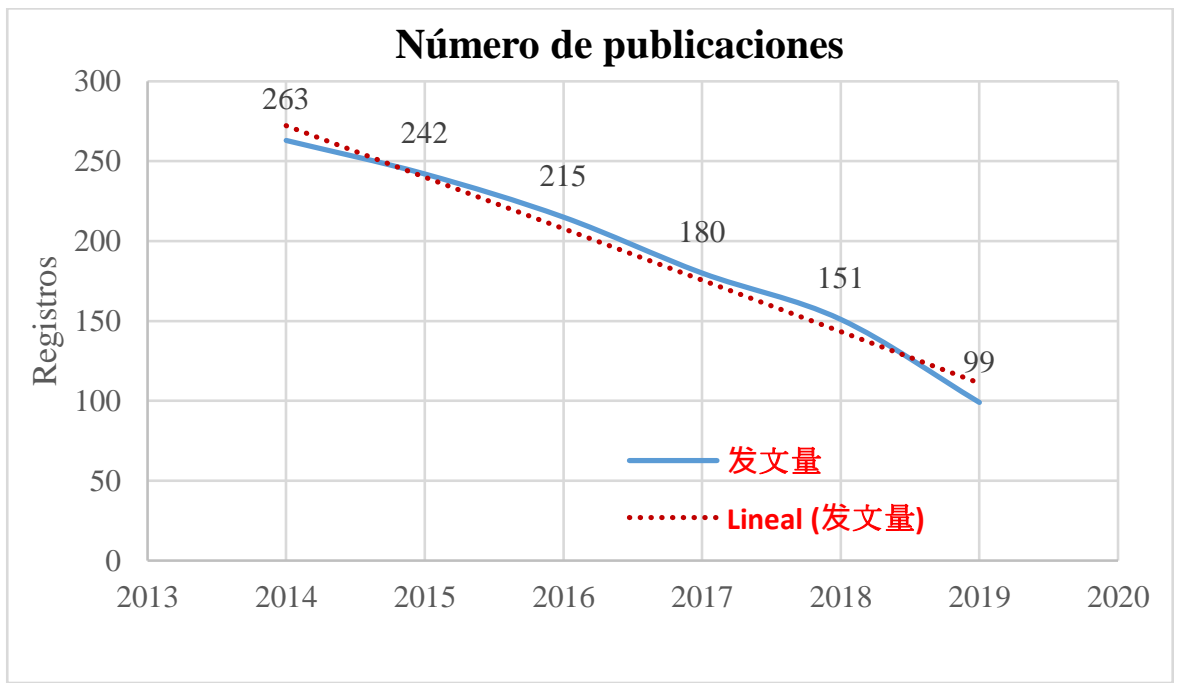

Figura 2. "Evolución del número de publicaciones desde 2013 hasta 2019"

Fuente: Elaboración propia

* línea azul es el número de publicaciones, la roja es la línea de tendencia lineal.

Derek J. de Solla Price (1956) constató que el crecimiento de la información científica era exponencial y se producía a un ritmo tan rápido que cada 10-15 años la información global existente se duplicaba (ley de Price) (Jordi A, 2012, p. 14) (en la figura 3). A su vez, propuso varias etapas: una fase de crecimiento exponencial propiamente dicha, en la que la tasa de crecimiento es proporcional al tamaño de la muestra, y una fase de crecimiento lineal, en la que la tasa de crecimiento es constante 0 independiente del tamaño del sistema (Tomás-Górriz $\mathrm{V}$ y Tomás-Casterá V, 2018, p. 148).

Las etapas son las siguientes:

- Fase 1: precursores

- Fase 2: crecimiento exponencial

- Fase 3: crecimiento lineal

- Fase 4: colapso del campo científico (saturación) 


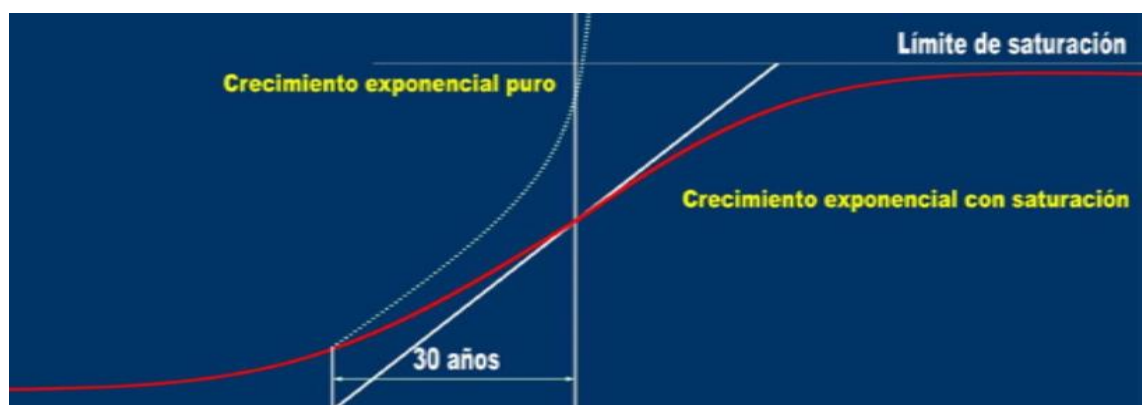

Figura 3. "Ley crecimiento exponencial de la ciencia" (D.J.S. Price)

Fuente: Tomás-Górriz V, Tomás-Casterá. La Bibliometría en la evaluación de la actividad científica. Hosp Domic. 2018;2(4):145-63.

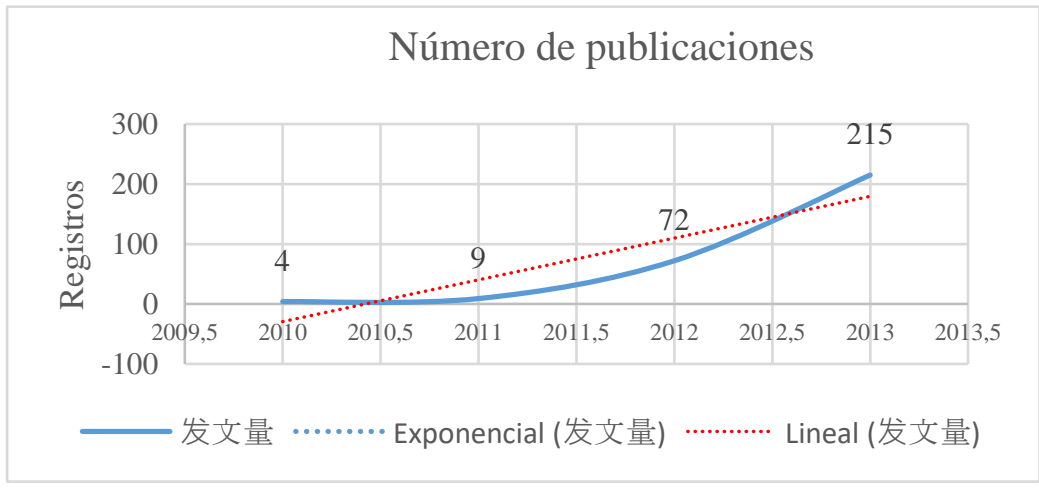

Figura 4. "Evolución del número de publicaciones desde 2009 hasta 2013"

Fuente: Elaboración propia

* línea azul es la del número de publicaciones. * * La puntada azul se trata de la línea de tendencia exponencial que se coincide con la azul, la puntada roja es la línea de tendencia exponencial.

La figura 4 muestra la situación de las publicaciones entre 2010 y 2013. Podemos observar que la puntada roja es la línea de tendencia exponencial y la línea azul representa el número de publicaciones, además, la línea de tendencia exponencial se coincide perfectamente con la línea de número de publicaciones.

Según la figura 2 y la figura 4, podemos concluir que, en cuanto a este estudio, entre 1999 y 2009 se desarrolla la fase 1, hay primeras publicaciones en el campo de investigación; entre 2010-2013 se encuentra la fase 2, 
crecimiento exponencial puro, desde Figura 2, la línea exponencial de pronóstico casi coincide con la de crecimiento; después de 2014 es la fase 4, el colapso del campo científico, donde se muestra un declive lineal. Todo nos presenta que la investigación del campo alcanzó el límite de crecimiento y sin un nuevo desarrollo literario o teórico, no avanzará considerablemente.

\subsection{Análisis de Palabras clave}

\subsubsection{Principales líneas de investigación}

- Distribución de nodos de palabras clave

Las palabras clave de los artículos representan su núcleo, si una palabra clave aparece muchas veces significa que el tema de investigación representado por este es una línea de investigación de campo (Feng y Wang, 2014). Hemos recurrido a las palabras clave de los artículos fuente de cada línea, seleccionando el más representativos mediante el algoritmo likelihood ratio. En la tabla 1, se resumen las palabras clave obtenidas a través de Citespace según la centralidad. Y la Tabla 2 muestra un ranking de las palabras clave según sus frecuencias. Según las dos tablas, podemos observar las líneas de investigación aproximadamente, pero el resultado no es tan satisfactorio.

\begin{tabular}{ccc}
\hline Rank & Centralidad & Palabra clave \\
\hline 1 & 0.32 & Ge Haowen \\
2 & 0.21 & La república del vino \\
3 & 0.2 & Mo Yan \\
4 & 0.18 & Escritor \\
5 & 0.18 & Ética en la traducción \\
6 & 0.16 & "going out" \\
7 & 0.16 & estrategia de traducción \\
8 & 0.13 & Grandes pechos, amplias caderas \\
9 & 0.11 & traducción en inglés \\
10 & 0.11 & El suplicio del aroma de sándalo \\
11 & 0.1 & poesía \\
12 & 0.1 & La República Popular China \\
13 & 0.09 & novelas de Mo Yan
\end{tabular}




\begin{tabular}{|c|c|c|c|c|}
\hline $\begin{array}{l}14 \\
15\end{array}$ & ola $1:$ Ni & $\begin{array}{r}0.09 \\
0.09 \\
\text { Imero de las } \\
\mathrm{Fl}\end{array}$ & $\begin{array}{l}5 \text { palab } \\
\text { ante: Ele }\end{array}$ & $\begin{array}{l}\text { traducción literaria } \\
\text { Adaptación } \\
\text { as clave de mayor centralidad } \\
\text { ooración propia }\end{array}$ \\
\hline Rank & Count & Centrality & Year & $\begin{array}{l}\text { Cited Rederence, palabras } \\
\text { clave }\end{array}$ \\
\hline 1 & 371 & 0,09 & 2006 & Mo Yan \\
\hline 2 & 289 & 0,13 & 2012 & Ge Haowen \\
\hline 3 & 126 & 0,1 & 2012 & Traducción \\
\hline 4 & 110 & 0,07 & 2012 & Estrategias de traducción \\
\hline 5 & 103 & 0,18 & 2012 & $\begin{array}{c}\text { La vida y la muerte me están } \\
\text { desgastando }\end{array}$ \\
\hline 6 & 79 & 0,09 & 2012 & El clan del sorgo rojo \\
\hline 7 & 68 & 0,16 & 2012 & Traducción literaria \\
\hline 8 & 67 & 0,06 & 2012 & Premio Nobel de Literatura \\
\hline 9 & 60 & 0,06 & 2009 & Escritor \\
\hline 10 & 52 & 0,06 & 2009 & Grandes pechos, amplias caderas \\
\hline 11 & 45 & 0,07 & 2014 & Novelas de Mo Yan \\
\hline 12 & 41 & 0,03 & 2013 & Marcadores culturales específicos \\
\hline 13 & 41 & 0,06 & 2012 & Literatura contemporánea china \\
\hline 14 & 40 & 0,06 & 2013 & Estudios de traducción \\
\hline 15 & 40 & 0,12 & 2013 & El clan del sorgo rojo \\
\hline 16 & 39 & 0,04 & 2013 & Subjetividad del traductor \\
\hline
\end{tabular}

Hikma 20 (1) (2021), 275 - 305 


$\begin{array}{ccccc}17 & 39 & 0,12 & 2013 & \text { Rana } \\ 18 & 38 & 0,08 & 2013 & \text { La república del vino } \\ 19 & 37 & 0,08 & 2013 & \text { Traducción en inglés } \\ 20 & 36 & 0,04 & 2012 & \text { Literatura } \\ 21 & 34 & 0,07 & 2012 & \text { Premio Nobel } \\ 22 & 31 & 0,09 & 2014 & \text { Métodos de traducción } \\ 23 & 30 & 0,08 & 2012 & \text { China } \\ 24 & 30 & 0,03 & 2014 & \text { Ideología } \\ 25 & 28 & 0,03 & 2014 & \text { Difusión }\end{array}$

Tabla 2: Resumen de las 25 palabras clave más citadas Fuente: Elaboración propia

Después de extraer las palabras clave, debemos tener en claro sus campos más relacionados y su evolución. La Figura 5 representa la visualización de las líneas temporales de clústeres cocitados de palabras clave en investigaciones sobre la traducción de obras de Mo Yan. Time-line view, es un tipo de representación que proporciona una visualización de la red por medio de líneas de tiempos horizontales.

Los nodos con mayor tamaño se traducen como mayor importancia de la base de publicaciones de las que se nutren los miembros de la especialidad (Olmeda-Gómez y Perianes-Rodríguez, 2019, p.7). Además, cada nodo representa un keyword, los enlaces entre nodos representan las relaciones cocitadas, los enlaces más gruesos significan que las relaciones entre nodos son más estrechas y crecen sus posibilidades de aparecer en un mismo artículo (Chen, 2012; Chen et al., 2010; Chen et al., 2012).

En la figura podemos ver que el nodo de mayor tamaño es "Mo Yan" (con 371 veces); el segundo es "Ge Haowen" (con 289 veces); el tercero es "traducción" (con 126 veces); y los siguientes son: "estrategia de traducción" (110 veces), "La vida y la muerte me están desgastando" (103 veces), "El clan del sorgo rojo" (81 veces), "traducción literaria" (68 veces), "Premio Nobel de Literatura" (67 veces). Además, se ha dividido en periodos. Mediante la 
visualización en una línea de tiempo, podemos identificar la evolución de cada nodo, cada término más relevante.

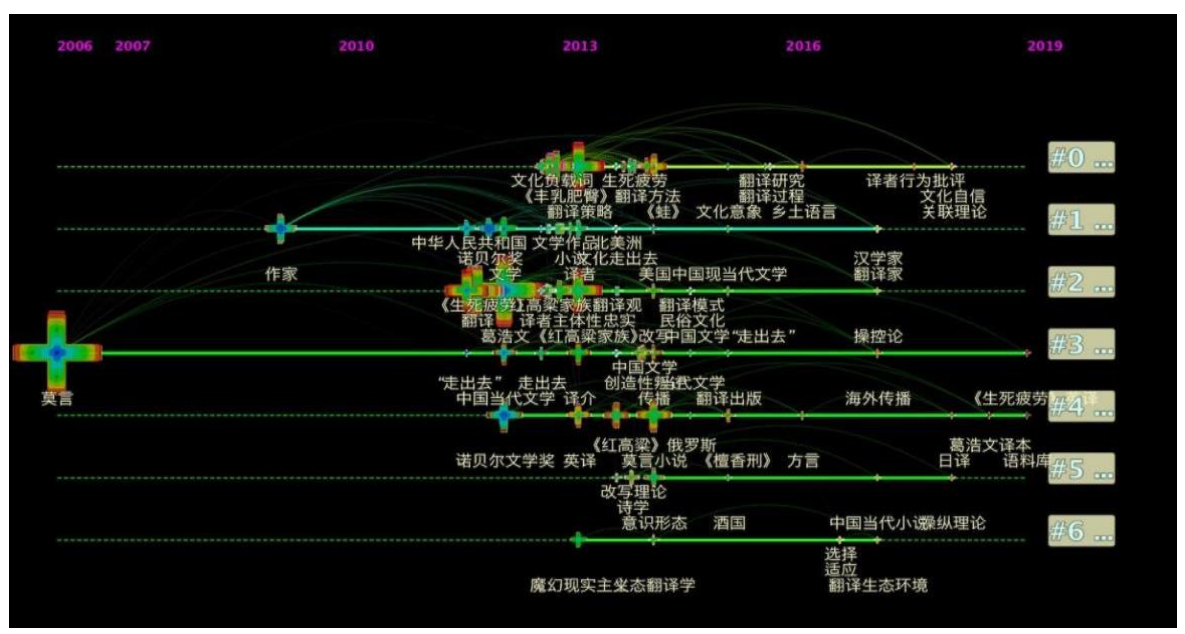

Figura 5" Time-line view de los clústeres" Fuente: Elaboración propia

Generalmente, las 8 palabras clave representan 8 especialidades de la investigación de la traducción de obras de Mo Yan durante casi 20 años.

"Ge Haowen" aparece por primera vez en 2012, y los nodos que se relacionan con esta búsqueda son: "El clan del sorgo rojo", "estrategia de traducción", "traducción literaria", "la vida y la muerte me están desgastando", "literatura contemporánea china", "Premio Nobel de Literatura". En 2013, fueron "Castigo de sándalo", "subjetividad del traductor"3, "Grandes pechos, amplias caderas", "Rana", "la república del vino", "marcador cultural específico"4.

${ }^{3}$ El traductor es el tema de la traducción y un participante importante en la construcción de la cultura nacional. Sin embargo, el tema de la traducción ha estado cubierto durante mucho tiempo en el sistema multicultural de la cultura china y ha aparecido la marginación del estado cultural de los traductores.

${ }^{4}$ Marcador Cultural Específico, que coincide con el que Herrero Rodes (1999) utiliza en su tesis doctorado, la definición es: Aquellos elementos que actualizados en el texto origen suponen un problema de traducción en el proceso de transferencia concreto por carecer la lengua meta de un término capaz de denotar, connotar y funcionar como el original, o simplemente porque el referente que designa no existe (Herrero Rodes, 1999: 139).

Hikma 20 (1) (2021), 275 - 305 
"Traducción" se relaciona estrechamente con "escritor", "La vida y la muerte me están desgastando", "El clan del sorgo rojo", "traducción literaria", "marcadores culturas específicos", "Premio Nobel de Literatura", "novelas de Mo Yan".

"Estrategia de traducción" se vincula a "métodos de traducción", "Sorgo Rojo", "traducción literaria", "traducción en inglés", "subjetividad del traductor", etcétera.

"La vida y la muerte me están desgastando", este nodo tiene relación con las palabras clave como "novelas de Mo Yan", "Sorgo rojo", "traducción literaria", "obras literarias", "traducción en inglés", "subjetividad del traductor".

"El clan del sorgo rojo" está relacionado con "traducción literaria", "estrategia de traducción", "obras literarias", "traducción en inglés", "subjetividad del traductor".

Y "traducción literaria" con "estrategias de traducción", "traductor", "la compensación estrategia de traducción", "técnicas de traducción", "creative treason de traducción", etcétera.

\begin{tabular}{|c|c|c|c|c|}
\hline & 1 & 2 & 3 & 4 \\
\hline & Ge Haowen & Traducción & $\begin{array}{l}\text { Estrategia de } \\
\text { traducción }\end{array}$ & $\begin{array}{c}\text { La vida y la muerta } \\
\text { me están } \\
\text { desgastando }\end{array}$ \\
\hline & 5 & 6 & 7 & 8 \\
\hline & El clan del sorgo & Traducción & Premio Nobel de & Literatura \\
\hline 2012 & rojo & literaria & Literatura & $\begin{array}{c}\text { contemporánea } \\
\text { china }\end{array}$ \\
\hline & 9 & 10 & 11 & 12 \\
\hline & Literatura & Premio Nobel & China & Novela \\
\hline & 13 & 14 & 15 & 16 \\
\hline & $\begin{array}{l}\text { Realismo } \\
\text { mágico }\end{array}$ & Sorgo Rojo & Traductor & Defamiliarization \\
\hline & 1 & 2 & 3 & 4 \\
\hline 2013 & $\begin{array}{l}\text { Traducción } \\
\text { literaria china }\end{array}$ & Jia Pingwa $^{5}$ & Hermeneutics & Crítica literaria \\
\hline
\end{tabular}

${ }^{5}$ Jia Pingwa, escritor de China, su obra maestra es FeiDu.

Hikma 20 (1) (2021), 275 - 305 


\begin{tabular}{|c|c|c|c|c|}
\hline & $\begin{array}{c}5 \\
\text { Estrategia de } \\
\text { compensación } \\
\text { de traducción }\end{array}$ & $\begin{array}{c}6 \\
\text { Gao Xingjian } 6\end{array}$ & $\begin{array}{c}7 \\
\text { Dream of Ding } \\
\text { Village }\end{array}$ & $\begin{array}{c}8 \\
\text { Shifu, harías } \\
\text { cualquier cosa por } \\
\text { divertirte }\end{array}$ \\
\hline \multirow{8}{*}{2014} & 1 & 2 & 3 & 4 \\
\hline & $\begin{array}{c}\text { Novelas de Mo } \\
\text { Yan }\end{array}$ & $\begin{array}{l}\text { Métodos de } \\
\text { traducción }\end{array}$ & Ideología & Comunicación \\
\hline & 5 & 6 & 7 & 8 \\
\hline & $\begin{array}{l}\text { Creative } \\
\text { Treason }^{7}\end{array}$ & Adaptación & Poesía & $\begin{array}{c}\text { La vida y la muerte } \\
\text { me están } \\
\text { desgastando }\end{array}$ \\
\hline & 9 & 10 & 11 & 12 \\
\hline & $\begin{array}{l}\text { Teoría de } \\
\text { adaptación }\end{array}$ & $\begin{array}{l}\text { Equivalencia } \\
\text { dinámica y } \\
\text { formal }\end{array}$ & Patrocinador & $\begin{array}{l}\text { Grandes pechos, } \\
\text { amplias caderas }\end{array}$ \\
\hline & 13 & 14 & 15 & 16 \\
\hline & $\begin{array}{l}\text { Literatura } \\
\text { mundial }\end{array}$ & Japón & Los EE. UU & Traductor \\
\hline \multirow{4}{*}{2015} & 1 & 2 & 3 & 4 \\
\hline & $\begin{array}{l}\text { "going out" de la } \\
\text { literatura china }\end{array}$ & $\begin{array}{c}\text { Literatura } \\
\text { contemporánea } \\
\text { china }\end{array}$ & $\begin{array}{l}\text { Literatura } \\
\text { contemporánea }\end{array}$ & $\begin{array}{l}\text { Modelo de } \\
\text { traducción }\end{array}$ \\
\hline & 5 & 6 & 7 & 8 \\
\hline & $\begin{array}{l}\text { Imaginación } \\
\text { cultural }\end{array}$ & $\begin{array}{l}\text { Cultura } \\
\text { folklórica }\end{array}$ & $\begin{array}{c}\text { La } \\
\text { compensación } \\
\text { estrategia de } \\
\text { traducción }\end{array}$ & $\begin{array}{c}\text { La república del } \\
\text { vino }\end{array}$ \\
\hline \multirow[b]{2}{*}{2016} & 1 & 2 & 3 & 4 \\
\hline & Dialectos & Dialectos & Sinólogo & $\begin{array}{c}\text { Teoría de la } \\
\text { relevancia }\end{array}$ \\
\hline
\end{tabular}

${ }^{6}$ Gao Xingjian, escritor en lengua china, dramaturgo y novelista, su novela más importante es $L a$ Montaña del alma.

${ }^{7}$ Una teoría en la traducción.

${ }^{8}$ La estrategia de "going out" de la literatura china es la política de construcción cultural propuesta por China a principios del siglo XXI. 


\begin{tabular}{|c|c|c|c|c|}
\hline & $\begin{array}{c}5 \\
\text { Modelo de } \\
\text { traducción }\end{array}$ & $\begin{array}{c}6 \\
\text { Toury }\end{array}$ & $\begin{array}{l}\quad 7 \\
\text { Proceso de } \\
\text { traducción }\end{array}$ & $\begin{array}{c}8 \\
\text { Investigación de } \\
\text { Mo Yan }\end{array}$ \\
\hline \multirow{4}{*}{2017} & 1 & 2 & 3 & 4 \\
\hline & Corpus & $\begin{array}{l}\text { Ambiente } \\
\text { ecológico de } \\
\text { traducción }\end{array}$ & $\begin{array}{l}\text { Translator } \\
\text { Behavior } \\
\text { Criticism }^{10}\end{array}$ & Giro cultural ${ }^{11}$ \\
\hline & 5 & 6 & 7 & 8 \\
\hline & $\begin{array}{l}\text { Novelas } \\
\text { contemporáneas } \\
\text { chinas }\end{array}$ & $\begin{array}{l}\text { Teoría de } \\
\text { manipulación }\end{array}$ & $\begin{array}{l}\text { Traducción } \\
\text { literaria }\end{array}$ & Adaptación \\
\hline \multirow{4}{*}{2018} & 1 & 2 & 3 & 4 \\
\hline & $\begin{array}{l}\text { Confianza de } \\
\text { cultura }\end{array}$ & $\begin{array}{l}\text { Traducción en } \\
\text { japonesa }\end{array}$ & $\begin{array}{c}\text { Teoría de } \\
\text { manipulación }\end{array}$ & Mo Yan Lun \\
\hline & 5 & 6 & 7 & 8 \\
\hline & $\begin{array}{l}\text { El rábano } \\
\text { transparente }\end{array}$ & Comunicación & $\begin{array}{c}\text { Yasunari } \\
\text { Kawabata }^{12}\end{array}$ & $\begin{array}{l}\text { Traducción de } \\
\text { humor }\end{array}$ \\
\hline \multirow{4}{*}{2019} & 1 & 2 & 3 & 4 \\
\hline & $\begin{array}{c}\text { Traducciones de } \\
\text { Ge Haowen }\end{array}$ & Intertextualidad & $\begin{array}{l}\text { Investigación de } \\
\text { novelas }\end{array}$ & $\begin{array}{l}\text { Características de } \\
\text { género literario }\end{array}$ \\
\hline & 5 & 6 & 7 & 8 \\
\hline & $\begin{array}{l}\text { Conceptos de } \\
\text { traducción }\end{array}$ & $\begin{array}{c}\text { la } \\
\text { Compensación } \\
\text { estrategia de } \\
\text { traducción }\end{array}$ & Contextualization & Capital \\
\hline
\end{tabular}

Tabla 3: Las palabras clave entre 2012-2019

Fuente: Elaboración propia

\footnotetext{
${ }^{9}$ Gideon Toury fue un erudito de traducción israelí y profesor de Poética, fue un pionero de los estudios de traducción descriptiva.

10 Translator Behavior Criticism。

${ }^{11}$ Esta idea comenzó a gestarse a finales del siglo XX por Sussan Bassett y André Lefevere

12 Yasunari Kawabata, escritor-novelista, fue el primer japonés en ganar el Premio Nobel de Literatura en 1968.
} 
La Figura 5 muestra diferentes nodos distribuidos a lo largo de un año (1999-2020). La Tabla 3 presenta las palabras clave de cada año.

Después de que Mo Yan ganara el Premio Nobel de Literatura, el escritor recibió repentinamente mucha atención. Además, Ge Haowen, sinológico y traductor estadounidense de renombre, tradujo El clan del Sorgo Rojo. Esta obra atrajo mucha admiración y ayudó a Mo Yan a ser famoso de la noche a la mañana. La otra obra importante traducida por Ge Haowen es La vida y la muerte me están desgastando. A partir de ahí, "Ge Haowen", "El clan de Sorgo Rojo", "La vida y la muerte me están desgastando" y "Premio Nobel" se alzaron como términos clave de los investigadores en 2012.

En 2013, lo lograron dos secuencias más llamativas: "Jia Pingwa" y "Gao Xingjian". El último ganó el Premio Nobel en 2000, y también se convirtió en un escritor de trascendencia internacional. En oposición a Xingjian, Jia Pingwa es un representante del movimiento de Xungen o la literatura de las raíces ${ }^{13}$. Eso supone que algunos investigadores chinos ya le prestaban más atención que a otros escritores; sobre todo, a los destacados del movimiento de Xungen.

En 2014, el número de los artículos sobre este tema llegó a su cenit, y el foco de investigación se centró en buscar "métodos de traducción" e "ideología". No obstante, "teoría de adaptación", "equivalencia dinámica y formal" y "otras teorías" empezaban a aplicarse en la investigación de la traducción de las obras del ganador. Salvo los cambios teóricos, la obra traducida que llamó más la atención durante ese año fue Grandes pechos, amplias caderas. Cabe destacar que muchos autores comenzaban a investigar la situación general de las obras traducidas en Japón. En 2015, el lema "la literatura china going out" se hacía eco, lo que indica que la la literatura china se inclinaba a creer que el mundo quería escuchar cuentos chinos.

Entre 2016 y 2017, una vez fijado el corpus, las técnicas planteadas por éste se empezaron a aplicar gradualmente en la investigación de las obras traducidas de Mo Yan. Además, las búsquedas de "dialectos" y el "ambiente ecológico de traducción" aumentaban. Sin embargo, entre 2018 y 2019, seguían ganando las búsquedas sobre teorías de traducción.

Con la muestra de las palabras de mayor frecuencia (Tabla 2) y su centralidad (Tabla 1), sabemos que durante los 20 años, las líneas de investigación se concentran principalmente en los temas siguientes: "Mo

\footnotetext{
${ }^{13}$ La literatura de las raíces se define la forma literaria tomando "buscando raíces culturales". A mediados de la década de los 80 del siglo 20 , los escritores chinos empiezan a dedicarse a explorar la conciencia tradicional y la cultura nacional.
} 
Yan"; "Ge Haowen"; Estrategias de traducción; "La vida y la muerte me están desgastando", "El clan del sorgo rojo"; " Traducción literaria"; "Escritor"; "going out" de la literatura china; "Grandes pechos, amplias caderas"; y "La república del vino".

\subsection{Análisis de clústeres de time-line}

Para profundizar una aproximación de los temas generales de la traducción de las obras de Mo Yan, hay que analizar los clústeres de las palabras clave. La red de Minimum Spanning Tree se ha descompuesto en 10 clústeres. La descomposición ha dado un valor global de modulalidad $(Q=0.5071)$. La modularidad mide el grado en que la red puede descomponerse en componentes menores (módulos) (Olmeda-Gómez, C; Perianes-Rodríguez, A, 2019). El valor indica que la red ha sido dividida razonablemente bien. Además, la calidad interna de configuración de los clusteres es buena, con un valor medio de silueta de 0.76 .

En la tabla 4 aparecen los 7 clústeres y los 4 sintagmas nominales seleccionados por log-likelihood ratio. Además del tamaño medido por el número de trabajos, aparece el año promedio de las fechas de publicación de los trabajos integrados en cada clúster cocitado.

Desde la tabla 4, concluimos que desde 1999, los temas generales de la investigación se dividen en 7 campos: clúster\# 0 , investigación sobre la traducción de los marcadores culturales específicos; clúster\# 1, "going out" de los escritores chinos y de la literatura china; clúster\# 2, investigación sobre el traductor Ge Haowen, sobre todo consideraciones en Sorgo Rojo y las estrategias de traducción; clúster\# 3, la aceptación de las obras traducidas; clúster\# 4, la transmisión de la literatura contemporánea china y la publicación de las ediciones de traducción de obras contemporáneas; clúster\# 5, poesía e ideología; y clúster\# 6, Ecología de la traducción, translational ecoenvironment.

\begin{tabular}{|c|c|c|c|c|}
\hline № ID & Tamaño & Silueta & Año & Términos top log-likehood ratio \\
\hline 0 & 24 & 0.691 & 2013 & $\begin{array}{l}\text { marcadores culturales específicos; } \\
\text { teoría de relevancia;Toury; métodos de } \\
\text { traducción }\end{array}$ \\
\hline 1 & 24 & 0.737 & 2014 & $\begin{array}{l}\text { obras literarias; lectores; China; } \\
\text { literatura }\end{array}$ \\
\hline 2 & 20 & 0.593 & 2014 & $\begin{array}{l}\text { Sorgo Rojo;Ge Haowen; } \\
\text { domesticación; extranjerización }\end{array}$ \\
\hline 3 & 16 & 0.727 & 2014 & $\begin{array}{l}\text { "going out "de cultura; aceptación; } \\
\text { estudios en traducción }\end{array}$ \\
\hline
\end{tabular}

Hikma 20 (1) (2021), 275 - 305 


$\begin{array}{ccccc}4 & 20 & 0.713 & 2013 & \begin{array}{c}\text { obras de Mo Yan; estrategia de } \\ \text { traducción; Sorgo Rojo;edición de } \\ \text { traducción por Ge Haowen }\end{array} \\ 5 & 8 & 0.898 & 2013 \begin{array}{c}\text { poesía; patrocinador; ideología; el } \\ \text { concepto de reescritura } \\ \text { elección; adaptación; ecología de } \\ \text { traducción; translational eco- } \\ \text { environment }\end{array}\end{array}$

Tabla 4: Resumen de los mayores clusteres de co-ocurrencia de palabras clave apartir de Pruning: Minimum Spanning Tree

Fuente: Elaboración propia

Los clusteres\#0 y \#1 son de mayor tamaño, además, los años de publicación relativamente son 2013 y 2014 . Esto supone que los temas de los dos son principales y los dos clústeres obtienen más número de artículos. Los clústeres \#2 y \#4 tienen el tamaño 20, el número de trabajo ocupa el segundo puesto.

El valor de la silueta mide la homogeneidad de un clúster (OlmedaGómez y Perianes-Rodríguez, 2019, p. 12). Si el valor es más alto, la homogeneidad es más alta. El valor de $S$ de los clústeres \# 3,4,5,6 es más de 0.7 , entre ellos, el cluster\# 5 con un valor de silueta muy elevado de 0.898 y el cluster \# 6, 0.961, que designa la coherencia de ítem que lo integran. Esto sugiere que el conjunto asociado de estos clústeres se corresponde de forma aproximada con la especialidad. En comparación con los 4, el clúster\# 2 y el clúster\# 0 tienen el valor de silueta relativamente bajo, esto significa que los elementos tienen la menor similitud.

\subsection{Análisis de citation burst}

\subsubsection{Cambios y Fronteras de Investigación}

Una citation burst ('explosión de citas') en CiteSpace significa que la frecuencia de utilización de las frases o palabras aumenta repentinamente (Ran y Zhang, 2016). Un burst se produce cuando una variable cambia mucho dentro de un período corto; esta variable puede ser la frecuencia de una palabra o de una frase en documentos citados, también puede ser el documento citado, el título, el autor, etc. (Chen, 2012, p.21). En comparación con palabras clave, un citation burst refleja más concretamente las características dinámicas, así que, esta muestra exactamente las fronteras de investigación (Li, 2014). No obstante, citation burst consiste en nodos de número de citaciones que suben o bajan bruscamente, este tipo de nodo normalmente representa el cambio en la dirección de una investigación (Chen 
yue, Chao mei chen, 2014:88). Por eso, si analizamos los citation burst de los registros se puede observar cuáles fueron los cambios en la investigación de las obras traducidas de Mo Yan durante los 20 años estudiados.

Figura 7 indica la exposición de citas del trabajo. Según los datos de CiteSpace, podemos ver las 8 citas más buscadas (figura 7). Ellas son "Grandes pechos, amplias caderas" (2014-2015), "Mo Yan" (2006-2013), "Premio de Nobel", "China", "Premio Nobel de Literatura" (2012-2013), "Rana" (2017-2020), "escritor" (2009-2013), "marcadores culturales" (2017-2018). Se puede considerar que entre 2012 y 2013 aparecieron decenas de artículos sobre el Premio Nobel de Literatura; entre 2014 y 2015, las búsquedas se centraron en información sobre Grandes pechos, amplias caderas, y podemos deducir que esta obra creó una tendencia emergente; entre 2017 y 2018, "marcadores culturales" atrajo un alto grado de atención; y, desde 2017 hasta ahora Rana es el nodo de mucha actividad y tendencia futura.

\begin{tabular}{|c|c|c|c|c|c|c|c|c|c|c|}
\hline \multicolumn{11}{|c|}{ CitationBurstHistory } \\
\hline keyword & TC & Centrality & Burst & PY & BurstBegin & BurstEnd & Span & HalfLife2 & Status & WaitingTime \\
\hline @PHRASE丰乳肥筲 & 10.0 & $5.64049698999198 \mathrm{E}-04$ & 3.2365 & 2014 & 2014 & 2015 & 2 & 1.0 & 1 & 0 \\
\hline @PHRASE莫言 & 371.0 & 0.22863915062893000 & 9.5012 & 2006 & 2006 & 2013 & 8 & 9.0 & 1 & 0 \\
\hline @PHRASE诺贝尔奖 & 34.0 & 0.02489398182980100 & 8.5639 & 2012 & 2012 & 2013 & 2 & 1.0 & 1 & 0 \\
\hline @PHRASE中华人民共和国 & 30.0 & 0.06023094289976990 & 3.6495 & 2012 & 2012 & 2013 & 2 & 2.0 & 1 & 0 \\
\hline @PHRASE诺贝尔文学奖 & 67.0 & 0.0619325068134861 & 11,8624 & 2012 & 2012 & 2013 & 2 & 1.0 & 1 & 0 \\
\hline @PHRASE 《蛙》 & 39.0 & 0.09878218975984510 & 5.0098 & 2013 & 2017 & 2020 & 4 & 4.0 & 1 & 4 \\
\hline @PHRASE作家 & 60.0 & 0.0783414273596683 & 11.3916 & 2009 & 2009 & 2013 & 5 & 4.0 & 1 & 0 \\
\hline @PHRASE文化负载词 & 41.0 & 0.01870372717470940 & 3.5412 & 2013 & 2017 & 2018 & 2 & 4.0 & 1 & 4 \\
\hline
\end{tabular}

Figura 7 "Los términos con mayor citation burst" Fuente: Elaboración propia

\subsection{Análisis de instituciones}

Para aproximar la base de investigación, se analizaban los datos utilizando la base de CNKI, la tabla 5 ilustra las 15 instituciones chinas con más publicaciones. La institución con más publicaciones sobre la investigación de obras traducidas de Mo Yan es la Universidad de Estudios Internacionales de Shanghái, seguida por la Escuela de idiomas extranjeros de la Universidad de Shangdong, la Escuela de idiomas extranjeros de la Universidad de Estudios Internacionales de Beijing y la Escuela de idiomas extranjeros de la Universidad de Estudios Internacionales de Nanjing. Desde un punto de vista regional, la distribución de las instituciones está desequilibrada. Shanghái, Jinan, Beijing, Yangzhou las cuatro ciudades tiene más publicaciones, otras no. 


\begin{tabular}{|c|c|c|}
\hline Rank & $\begin{array}{l}\text { Número de } \\
\text { artículos }\end{array}$ & Instituciones \\
\hline 1 & 43 & Universidad de Estudios Internacionales de Shanghái \\
\hline 2 & 30 & Escuela de idiomas extranjeras de la Universidad de Shandong \\
\hline 3 & 30 & Universidad de Estudios Internacionales de Beijing \\
\hline 4 & 28 & Escuela de idiomas extranjeras de la Universidad de Nanjin \\
\hline 5 & 27 & $\begin{array}{l}\text { Escuela de idiomas extranjeras de la Universidad Normal del } \\
\text { Este de China }\end{array}$ \\
\hline 6 & 24 & Universidad de Yangzhou \\
\hline 7 & 22 & Universidad Normal de Shandong \\
\hline 8 & 21 & Universidad de Jilin \\
\hline 9 & 19 & Universidad Normal de Shaanxi \\
\hline 10 & 19 & Universidad de Suzhou \\
\hline 11 & 19 & Universidad Normal del Capital \\
\hline 12 & 18 & Universidad de Estudios Extranjeros de Guangdong \\
\hline 13 & 17 & Universidad Normal de Fujian \\
\hline 14 & 17 & Universidad Normal de Jiangxi \\
\hline 15 & 15 & Universidad Normal del Centro de China \\
\hline 16 & 15 & Universidad de Estudios Internacionales de Xi'an \\
\hline 17 & 15 & Universidad Normal de Beijing \\
\hline
\end{tabular}

Tabla 5: Instituciones por número de publicaciones Fuente: Elaboración propia

Hikma 20 (1) (2021), 275 - 305 


\subsection{Análisis de red de co-citación de autores}

\subsubsection{Situación de la cantidad de publicaciones}

En la Tabla 6 se puede observar que los 3 autores más prolíficos son Shao Lu, Zhou Lingshun, Wang Ning. Esto supone que son los autores más productivos en toda China. Después de leer sus artículos, sabremos que Shao Lu centró sus investigaciones en la traducción de La vida y la muerte me están desgastando, incluyendo sus paráfrasis y los Models of Translation Reading. Zhou Lingshun hizo análisis sobre la traducción a otros dialectos de las obras de Mo Yan y Translator Behavior Criticism. Wang Ning se inclinaba hacia los estudios de transculturalismo. No obstante, Ning Ming prestaba más atención a la aceptación de las obras traducidas de Mo Yan en el extranjero y a la libertad de sus novelas. Zhang Zhizhong hizo un recorrido de la historia de la averiguación de las obras de Mo Yan, al mismo tiempo, realizó varios apuntes sobre la relación entre el Premio Nobel de Literatura y China; Jiang Zhiqin analizó la difusión de las obras de Mo Yan en el extranjero; Wang Wenqiang estudió la situación general de las obras de Mo Yan desde el punto de vista de la teoría de Translation Ethics; y Liu Yunhong estudiaba la traducción de literatura china en general.

Índice $\mathrm{h}$ es un sistema para la medición de la calidad profesional tanto de físicos como de otros científicos. Un científico tiene un índice $h$, si el h de sus Np de trabajo recibe al menos h citas cada uno, y los otros (Np-h) trabajos tienen como máximo $\mathrm{h}$ citas cada uno ${ }^{14}$. Si un investigador tiene el índice $\mathrm{h}$ más alto, significa que él obtiene mayor importancia e influencia. Por ello, se observa que los autores han realizado estudios de diferentes temas desde varias perspectivas, sin embargo, todavía falta un investigador de influencia.

\begin{tabular}{cccccc}
\hline \multirow{2}{*}{ Rank } & Autores & $\begin{array}{c}\text { Número } \\
\text { de } \\
\text { artículos }\end{array}$ & $\begin{array}{c}\text { Frecuencia de Citación por } \\
\text { citaciones }\end{array}$ & artículo & Índice de H \\
\hline 1 & Shao Lu & 12 & 619 & 51,58 & 7 \\
2 & Zhou Lingshun & 11 & 162 & 14,72 & 7 \\
3 & Wang Ning & 9 & 382 & 42,44 & 4 \\
4 & Ning Ming & 8 & 85 & 10,63 & 3 \\
5 & Zhang & 8 & 31 & 3,88 & 3 \\
\hline
\end{tabular}

14 https://web.archive.org/web/20090124000049/http://pda.physorg.com/lofi-news-hindex-sayspapers $7971 . \mathrm{htm}$ 


\begin{tabular}{|c|c|c|c|c|c|}
\hline 6 & Jiang Zhiqin & 6 & 73 & 12,17 & 3 \\
\hline 7 & $\begin{array}{c}\text { Wang } \\
\text { Wenqiang }\end{array}$ & 6 & 67 & 11,17 & 5 \\
\hline 8 & Liu Yunhong & 6 & 243 & 40,5 & 4 \\
\hline 9 & Zhu Zhenwu & 5 & 37 & 7,4 & 2 \\
\hline 10 & Xu Jun & 5 & 275 & 55 & 5 \\
\hline 11 & Song Qingwei & 5 & 39 & 7,8 & 2 \\
\hline 12 & Liu Keqiang & 5 & 11 & 2,2 & 2 \\
\hline 13 & Zhang Jiguang & 5 & 61 & 12,2 & 4 \\
\hline
\end{tabular}

Tabla 6: Top 13 autores con más de 5 artículos

Fuente: Elaboración propia

\subsubsection{Análisis de autores principales y equipo de autores}

Según Price Law ${ }^{15}$, la mitad de los artículos científicos se han escrito por autores prolíferos, y el número equivale aproximadamente a la raíz cuadrada de la totalidad de autores (Kong, 2019, p.18). La fórmula es $\mathrm{Mp}=0.749 \sqrt{\mathrm{N}_{\max }}$. Según la tabla 4 , el mayor número de publicaciones por autor es 12 , de ahí tendríamos que $\operatorname{Nmax}=12$, por lo que el cálculo sería $M p=0.749 \sqrt{ } 12=2,59 \approx 3$, y esto significa que un autor con más de 3 artículos se puede considerar como el autor principal.

La cifra total de los autores principales es de 40 con 184 artículos, y esta cifra ocupa el $12,09 \%$ de la totalidad de artículos. Sin embargo, el valor estándar de Price Law es del $50 \%$, y nuestro $12,9 \%$ es muy inferior al $50 \%$. Esto supone que en China la investigación de obras traducidas de Mo Yan todavía no puede formar un grupo de investigadores claves.

En la figura 8 se muestra el análisis de co-citación de autores. En ella podemos ver la red de colaboración, co-citación y el grado de colaboración. A través de CiteSpace, se sacan $N=93, E=14$, la densidad $=0,0033$, $\mathrm{Q}=0.9337$, el nodo más grande representa la reiteración de apariciones. $\mathrm{Si}$ en la figura 4 observamos los tres nodos sobresalientes (que son los que resaltan), el mayor pertenece a Zhu Zhenwu, el segundo a Li Jingduan, y el último a Liu Meng.

\footnotetext{
${ }^{15}$ Price law mide la distribución de los autores en todas las disciplinas.
} 
Además, se ve claramente que los enlaces entre diferentes nodos son débiles, lo que significa que la investigación está descentralizada, y las relaciones y colaboraciones entre diferentes grupos son muy pocas.

\section{柳萌}

朱振武

李景端

Figura 8: Representación de red de co-citación de autores.

Fuente: Elaboración propia

Los tres nodos desde arriba abajo son: Liu Men, Zhu Zhenwu, Li Jinduan.

Hikma 20 (1) (2021), 275 - 305 
3.7. Análisis de las revistas con más publicaciones

\begin{tabular}{|c|c|c|}
\hline Rank & Revistas & Fre \\
\hline 1 & Contemporany Writers Review & 24 \\
\hline 2 & Overseas English & 24 \\
\hline 3 & Comentaris de Novelas & 19 \\
\hline 4 & EST Learning & 13 \\
\hline 5 & Chinese Translators Journal & 12 \\
\hline 6 & Comparative Literature in China & 11 \\
\hline 7 & Literature Press & 11 \\
\hline 8 & Literatures in Chinese & 10 \\
\hline 9 & Southern Cultural Forum & 9 \\
\hline 10 & Journal of Literature and Art & 9 \\
\hline 11 & Journal of Weifang University & 9 \\
\hline 12 & Foreign Lanuage and Literature & 9 \\
\hline 13 & China Reading Weekly & 9 \\
\hline 14 & Foreign Language Education & 8 \\
\hline 15 & A Vast View on Publishing & 7 \\
\hline 16 & Publishing Research & 7 \\
\hline 17 & Data of Culture and Education & 6 \\
\hline 18 & Modern Literary Magazine & 6 \\
\hline 19 & Democratic Consultative Newspaper & 6 \\
\hline 20 & MasterPieces Review & 6 \\
\hline 21 & Journal of Mudanjiang College of Education & 6 \\
\hline 22 & Journal of Chifeng University & 6 \\
\hline
\end{tabular}

Tabla 7: Revistas top de publicaciones en "traducción de obras de Mo Yan" 1999-2019

Fuente: Elaboración propia

Hikma 20 (1) (2021), 275 - 305 
La tabla 7 muestra las revistas con más de 6 publicaciones sobre el mismo tema, encontramos 19 revistas, entre ellas, Comentarios de Novelas, Chinese Translators Journal, Comparative Literature in China, Literature in Chinese, Southern Cultural Forum, Publishing Research que son del corpus de $\mathrm{CSSCl}^{16}$, además, Foreign Lanuage and Literature, Foreign Language Education son Core Journals Nacional. De este modo, se observa que salen muchos resultados de alta calidad de la investigación de las obras traducidas de Mo Yan.

\section{CONCLUSIONES}

En este estudio se ha analizado la situación general desde 1999 hasta el inicio de 2020 de la investigación sobre la traducción de las obras de Mo Yan, basándose en la construcción y el análisis de redes bibliográficas de cocitación de autores y palabras clave, además, teniendo en cuenta los datos de CNKI con el fin de conocer las líneas principales, los cambios de dirección, la tendencia futura, los autores principales, las instituciones, y las revistas principales y su desarrollo.

El análisis del grafo mostró que las palabras clave de la investigación son: "Mo Yan”, "Ge Haowen”, "estrategia de traducción”, "La vida y la muerte me están desgastando", "El Clan del sorgo rojo", "traducción literaria" y "Premio Nobel de Literatura". Estas palabras clave reflejan las líneas de investigación.

A través de la visualización de Time-line, sacamos las líneas de investigación según cada año. Y la tendencia de la investigación se concentra en las palabras clave siguientes: "intectualidad", "características de género literario", "conceptos de traducción”, “contextualización”, "capital”, etc.

Con el análisis de citation burst, deducimos que a lo largo de los 20 años estudiados: entre 1999 y 2012, la investigación de la traducción de las obras de Mo Yan avanzó muy poco, pero desde que recibió el Premio Nobel de Literatura, los estudios de traducción se desarrollaron a gran velocidad. En 2014, Grandes pechos, amplias caderas se convirtió en la obra más llamativa y en 2017, fue la obra la-más destacada.

En cuanto al análisis de las instituciones, las 5 instituciones con más publicaciones son: Universidad de Estudios Internacionales de Shanghái, Escuela de idiomas extranjeros de la Universidad de Shandong, Universidad de Estudios Internacionales de Beijing, Escuela de idiomas extranjeros de la Universidad de Nanjin, y Escuela de idiomas extranjeros de la Universidad Normal del Este de China. No obstante, concluimos que las instituciones

\footnotetext{
${ }^{16}$ CSSCI, Chinese Social Sciences Citation Index.
} 
están distribuidas principalmente en el suroeste de China y en la capital. Por lo que encontramos mucha distancia geográfica en la distribución de las instituciones.

Los 5 primeros autores de los artículos sobre este tema son: Shao Lu, Zhou Lingshun, Wang Ning, Ning Ming, y Zhang Zhizhong. Además, los trabajos se hicieron desde diferentes perspectivas y relativos a distintas obras y teorías. Pero aún faltan autores núcleo y las relaciones entre los grupos de investigación son muy escasas.

Las revistas Contemporany Writers Review, Overseas English, Comentarios de Novelas, EST Learning y Chinese Translators Journal han publicado numerosos trabajos sobre las traducciones de las obras de Mo Yan. Entre las revistas importantes de este campo de estudio, hay 8 revistas de CSSCI.

Con el cambio de la situación nacional e internacional, la investigación sobre la traducción de las obras de Mo Yan debería desarrollarse con mayor amplitud y profundidad. Para mí corresponde destacar también los aspectos siguientes:

1. Estudios de la traducción en otros idiomas y en los países de habla inglesa. Con el análisis de la situación general sabemos que la mayoría de las obras son traducidas en inglés, y la investigación de la traducción en otros idiomas es muy escasa, por ejemplo, la traducción al español, al alemán o al polaco. Los investigadores chinos deberían prestar más atención a los estudios de traducción en otros idiomas.

2. Estudios sobre el estilo y la subjetividad de los traductores de diferentes idiomas. Ahora el traductor más famoso de las obras de Mo Yan es Ge Haowen, sin embargo, el análisis de los traductores a lenguas extranjeras es muy limitado. Traductores como Blas Piñero Martínez ${ }^{17}$, Anne-Héléne Suárez Girard ${ }^{18}$, Karin Betz ${ }^{19}$, etc., son muy importantes y deberían prestarles más atención.

3. Estudios sobre otras obras de Mo Yan y otros escritores chinos. Actualmente, las obras más llamativas son El clan de sorgo rojo, Rana y La vida y la muerte me están desgastando, pero otras obras de Mo Yan como Cambios, ¡Boom! apenas están siendo estudiadas. Por último, además de Mo Yan, el otro escritor chino que aparece durante estos 20 años de investigación es Jia Pingwa,

\footnotetext{
${ }^{17}$ Traductor español de muchas obras de Mo Yan, por ejemplo: El manglar, El mapa del tesoro escondido, El suplicio de aroma de sándalo, etc.

${ }_{18}$ Traductora española de Cambios.

${ }^{19}$ Traductora alemana de El suplicio del aroma de sándalo.
} 
sin embargo, la investigación de otros escritores chinos importantes como Chen Zhongshi20, Wang Anyi ${ }^{21}$, Yan Lianke ${ }^{22}$, etc. se queda relativamente atrasada.

Al final, este artículo solo es una imagen acústica de la situación general de la investigación de traducción de las obras de Mo Yan en China mediante CiteSpace, esperamos más estudios y análisis mejores.

\section{REFERENCIAS BIBLIOGRÁFICAS}

Ardanuy Baró, Jordi. (2012). Breve Indroducción a la Bibliometría. Department de Biblioteconomia i Documentació, Universitat de Barcelona, Recuperado de http://diposit.ub.edu/dspace/bitstream/2445/30962/1/breve\%20introdu ccion\%20bibliometria.pdf

Borner, K., Chen, C. \& Boyack, K. (2003). Visualizing knowledge domains. In B. Cronin (Ed.), Annual Review of Information Science \& Technology, 37, pp.179-255.

Börner Katy., Chen Chaomei y Boyack Kevin. (2003). Visualizing Knowledge Domins. In Blaise Cronin (Ed.), Annual Review of Information Science \& Technology, Volume 37, Medford, NJ: Information Today, Inc/American Society for Information Science and Technology, chapter 5, pp. 179-255.

Chen, Yue., Chen, Chaomei., Hu, Zhigang., Wang, Xianwen. (2014). Principles and Applications of Analyzing a Citation Space. Science Press.

Chen, Chaomei. (2012). Predictive effects of structural variation on citation counts. Journal of and Technology, (3), 43-49.

Chen, Chaomei., Fidelia Ibekwe-Sanjuan y Hou Jianhua. (2010). The structure and dynamics of co-citation clusters: A multiple-perspective co-citation analysis. Journal of the American Society for Information Science and Technology, (7),1386-1409.

Chen, Chaomei., Hu, Zhigang., Liu, Shengbo y Hung, Tseng. (2012). Emerging trends in regenerative medicine: A scientometric analysis in CiteSpace. Expert Opinion onBiological Therapy. (5),593-608.

\footnotetext{
20 Escritor importante chino, representante del movimiento buscando raíces.

${ }^{21}$ Escritora china, obras representativas: Amor en un valle encantado, Amor en una colina desnuda...

${ }^{22}$ Escritor chino, obras representativas: El sueño de la aldea Ding, Crónica de una exposición...
} 
Ding, Y., Chowdhury, G., \& Foo, S. (2001). Bibliometric cartography of information retrieval research by using co-word analysis. Information Processing \& Management, 37(6), 817-842.

Feng, Jia 冯佳 y Wang, Kefei 王克非. (2014). 近十年国际语言规划和语言政策 研究的 CiteSpace 分析, 中国外语, 11(1), 69-84.

Freeman, Linton C. (1977). A set of measures on centrality based on betweenness. Sociometry, 40(1), 35-41.

Garfield E. (1955). Citation indices for science. Science, (122), 109-110.

Guerrero Chanduví, Dante A. M. y La Rosa Lama, Gerson; Panta Yenque, Manuel; Yagüe Blanco, José Luis. (2014, 16-18th july). Analysis of the Essential Literature on Project Management in Spanish. $18^{\text {th }}$ International Congress on Project Management and Engineering Alcañiz, 16-18 th July 2014, Piura, Perú.

Hyunjung, K. (2012). Examing the Knowledge Structure in the Communication Field: Author Cocitation Analysis for the Editorial Board of the Journal of Communication, 2008 and 2011. Journal of the Korean BIBLIA Society for library and Information Science, 23(2).

Koehler, W. (2001). Information science as "Little Science": The implications of a bibliometric analysis of the Journal of the American Society for Information Science. Scientometrics, 51(1),117-132. Recuperado de https://doi.org/10.1023/a:1010516712215

Kong, Zhen. (2019). 基于 CiteSpace 的国内隐喻研究计量学. Journal of Xi' an International Studies University, 27(4), 16-20.

Liu, Z., Yin, Y., Liu, W., \& Dunford, M. (2015). Visualizing the intellectual structure and evolution of innovation systems research: a bibliometric analysis. Scientometrics, 103(1), 135-158. Recuperado de https://doi.org/10.1007/s11192-014-1517-y

Luis Pinto, Adilson., Moreiro González, José Antonio., Gusmão, Alexandre Oliveira de Meira. (2009). Análisis de redes sociales a partir de recursos web y de bases de datos especializadas en literatura científica. ANALES DE DOCUMENTACION, (12), 139-158.

Martínez-Toro, G.A., Rico-Bautista, D., Romero-Riaño, E., Galeano-Barrera, C.J., Guerrero, Cesar D., Parra-Valencia, Jorge A. (2019). Análisis de la estructura intelectual y la evolución de la investigación en la interacción humano-computador: un análisis bibliométrico. Risti, 17(1), 363-378. 
Ketan K, Mane., Katy Börner. (2004). Mapping topics and topic bursts in PNAS. Proceedings of the National Academy of Sciences of the United States of America, v. 101, suppl 1. 5287-5290. Recuperado de https://doi.org/10.1073/pnas.0307626100

Naghizadeh, R., Elahi, S., Manteghi, M., Ghazinoory, S., \& Ranga, M. (2015). Through the magnifying glass: an analysis of regional innovation models based on co- word and meta-synthesis methods. Quality and Quantity, 49(6), 2481-2505. Recuperado de https://doi.org/10.1007/s11135-014-0123-7

Narin, F. (1987). Bibliometric techniques in the evaluation of research programs. Science and Public Policy, 14(2), 99-106. Recuperado de https://doi.org/10.1093/spp/14.2.99

Newman, Mark E. J. (2006). Finding community structure in networks using the eigenvectors of matrices. Physical review E, v. 74, n. 3,1-22. Recuperado de https://doi.org/10.1103/PhysRevE.74.036104

Olmeda-Gómez, C., Perianes-Rodríguez, A. (2019). Altmetría como especialidad de investigación (Dimensions, 2005-2018). El profesional de la información, 28(6), 1-19.

Rousseeuw, Peter J. (1987). A graphical aid to the interpretation and validtion of cluster analysis. Journal of computational and applied mathematics, (20), 53-65.

Sink, D. S., Tuttle, T. C., \& Devries, S. J. (1984). Productivity measurement and evaluation: What is available? National Productivity Review, 3(3), 265-287. Recuperado de_https://doi.org/10.1002/npr.4040030305

Small, H. (1973). Co-citation in the Scientific Literature: A New Measure of the Relationship between Two Documents. Journal of the American Society for Information Science and Technology, July-Augus, 265-269.

Terry, J. L. (1994). Authorship in College \& Research Libraries Revisited: Gender, Institutional Affiliation, Collaboration. College \& Research Libraries, 377-384.

Tomás-Górriz, V., y Tomás-Casterá, V. (2018). La Bibliometría en la evaluación de la actividad científica. Hosp Domic, 2(4), 145-163.

van Eck, N. J., \& Waltman, L. (2010). Software survey: VOSviewer, a computer program for bibliometric mapping. Scientometrics, 84(2), 523-538. Recuperado de https://doi.org/10.1007/s11192-0090146-3 
White, H. D., y Mccain, K. W. (1998). Visualizing a Discipline: An Author CoCitation Analysis of Information Science, 1972 - 1995. Society, 49(4), 327-355.

Wu, Yuntong.; Wang, Huijie.; Wang, Zhexiao.; Zhang,Bin.;Meyer Burghard C. (2020). Knowledge Mapping Analysis of Rural Landsape Using CiteSpace. Sustainability, 12(66), 1-17.

Zhen, Yuqian 郑羽菲. (2018). 莫言小说翻译研究现状综述. 科教导刊, 18(19). 